DRAFT VERSION OCTOBER 9, 2018

Preprint typeset using $\mathrm{LATEX}_{\mathrm{E}}$ style emulateapj v. 5/2/11

\title{
THE FORMATION OF SUPERMASSIVE BLACK HOLES FROM LOW-MASS POP III SEEDS
}

\author{
Daniel J. Whalen ${ }^{1}$ And Chris L. Fryer ${ }^{2}$ \\ Draft version October 9, 2018
}

\begin{abstract}
The existence of $10^{9} \mathrm{M}_{\odot}$ black holes $(\mathrm{BH})$ in massive galaxies by $z \operatorname{sim} 7$ is one of the great unsolved mysteries in cosmological structure formation. One theory argues that they originate from the black holes of Pop III stars at $z \sim 20$ and then accrete at the Eddington limit down to the epoch of reionization, which requires that they have constant access to rich supplies of fuel. Because early numerical simulations suggested that Pop III stars were $\gtrsim 100 \mathrm{M}_{\odot}$, the supermassive black hole seeds considered up to now were $100-300 \mathrm{M}_{\odot}$. However, there is a growing numerical and observational consensus that some Pop III stars were tens of solar masses, not hundreds, and that 20 - $40 \mathrm{M}_{\odot}$ black holes may have been much more plentiful at high redshift. However, we find that natal kicks imparted to 20 - $40 \mathrm{M}_{\odot}$ Pop III BHs during formation eject them from their halos and hence their fuel supply, precluding them from Eddington-limit growth. Consequently, supermassive black holes are far less likely to form from low-mass Pop III stars than from very massive ones.
\end{abstract}

Subject headings: black hole physics - cosmology: early universe - theory - galaxies: formation

\section{INTRODUCTION}

The existence of $10^{9} \mathrm{M}_{\odot}$ black holes $(\mathrm{BH})$ in massive galaxies by $z \sim 7$, only a billion years after the Big Bang (e.g. Mortlock et al. 2011), poses one of the great unsolved problems in cosmological structure formation. In the $\Lambda \mathrm{CDM}$ paradigm, early structure formation is hierarchical, with small objects at high redshifts evolving into ever more massive ones by accretion and mergers through cosmic time. For this reason it is generally supposed that the supermassive black holes (SMBH) that power the $z \sim 7$ Sloan Digital Sky Survey $(S D S S)$ quasars grow from much smaller seeds at earlier epochs. The origin of SMBH and how they reach such large masses in such short times is a subject of ongoing debate. Three modes of formation have been proposed for SMBH seeds: the collapse of Pop III stars into $100-300 \mathrm{M}_{\odot}$ black holes at $z \sim 20$ (Alvarez et al. 2009), baryon collapse in $10^{8}$ $\mathrm{M}_{\odot}$ dark matter halos that have somehow bypassed previous star formation into $10^{4}-10^{6} \mathrm{M}_{\odot}$ BH at $z \sim 15$ (Wise et al. 2008; Regan \& Haehnelt 2009; Shang et al. 2010), and more exotic pathways like the relativistic collapse of dense primeval star clusters into $10^{4}-10^{6} \mathrm{M}_{\odot}$ BH (see section 3.3 of Diorgovski et al. 2008, for a recent review).

Stellar-mass SMBH seeds form at $z \sim 20$ when Pop III stars die in either core-collapse supernovae (SNe, 15 - 45 $\left.\mathrm{M}_{\odot}\right)$ or by direct collapse to a BH $\left(45-100 \mathrm{M}_{\odot}, \gtrsim 260\right.$ $\mathrm{M}_{\odot}$ ) (Heger \& Woosley 2002). This formation channel is favored by some because most dark matter halos will form a Pop III star at this epoch if they reach masses of $\sim$ $10^{5} \mathrm{M}_{\odot}$ (Abel et al. 2002; Bromm et al. 2002). However, these $\mathrm{BH}$ have such low initial masses that they must continuously accrete at the Eddington limit to reach $10^{9}$ $\mathrm{M}_{\odot}$ by $z \sim 7$. This is problematic for several reasons. First, numerical simulations have shown that Pop III stars usually evaporate the halos that give birth to

\footnotetext{
${ }^{1}$ McWilliams Fellow, Department of Physics, Carnegie Mellon University, Pittsburgh, PA 15213

2 CCS-2, Los Alamos National Laboratory, Los Alamos, NM 87545
}

them, so the BH are 'born starving' (e.g. Whalen et al. 2004; Kitayama et al. 2004; Whalen et al. 2008b). Filamentary inflows and mergers later restore baryons to the halo but only after 50 - 100 Myr (Yoshida et al. 2007), during which crucial e-foldings in mass are lost. Second, preliminary studies indicate that once accretion commences, the $\mathrm{BH}$ itself emits ionizing radiation that disperses its own fuel supply, limiting its growth rate to a fraction of the Eddington limit (Milosavliević et al. 2009; Park \& Ricotti 2011, 2012) (but see Li 2011). Furthermore, if the seed $\mathrm{BH}$ is not confined to the halo, its duty cycle as it meanders through cosmological density fields is intermittent, which also curtails its growth (Alvarez et al. 2009).

Until now, $20-40 \mathrm{M}_{\odot}$ Pop III BH (Zhang et al. 2008; Whalen et al. 2008a) have been overlooked as candidates for SMBH seeds because previous studies assume that primordial stars are $\gtrsim 100 \mathrm{M}_{\odot}$. However, there is a growing numerical and observational consensus that some Pop III stars are tens of solar masses, not hundreds. More recent, much larger ensembles of numerical simulations found many halos with central collapse rates consistent with $20-60 \mathrm{M}_{\odot}$ for the final mass of the star (O'Shea \& Norman 2007) and that a fraction of the halos form binaries in this mass range (Turk et al. 2009). Furthermore, new simulations of the formation of Pop III protostellar accretion disks suggest that they were prone to fragmentation into as many as a dozen smaller stars (Stacy et al. 2010; Clark et al. 2011; Smith et al. 2011; Greif et al. 2011). Very preliminary calculations of Ifront breakout from these disks indicate that ionizing UV radiation may terminate accretion onto the nascent star at $\sim 40 \mathrm{M}_{\odot}$ (Hosokawa et al. 2011; Stacy et al. 2012).

On the observational side, recent attempts to reconcile the nucleosynthetic yields of Pop III supernovae with the chemical abundances found in ancient, dim extremely metal-poor stars in the Galactic halo suggest that 15 $40 \mathrm{M}_{\odot}$ primordial stars may have been responsible for most of the heavy elements expelled into the primeval IGM (Joggerst et al. 2010). The failure to detect the 
distinctive 'odd-even' nucleosynthetic signature of 140 $260 \mathrm{M}_{\odot}$ pair-instability $\mathrm{SNe}$ in metal-poor stars to date reinforces the fact that some Pop III stars might not be very massive, but this pattern may have been masked by selection effects in the observations (Karlsson et al. 2008).

Low-mass Pop III BH are crucially different from more massive $\mathrm{BH}$ because they are born in supernova explosions rather than by direct collapse. Asymmetries in the core-collapse engine can impart kicks of $200-1000 \mathrm{~km} / \mathrm{s}$ to $20-40 \mathrm{M}_{\odot} \mathrm{BH}$, ejecting them from the halos that gave birth to them. In this Letter we examine the implications of natal kicks for low-mass Pop III black holes as candidates for SMBH seeds. In $\S 2$ we review the formation pathways for low-mass Pop III BH. In $\S 3$ we calculate their post-supernova kinematics and retention fractions in halos. In $\S 4$ we conclude.

\section{LOW-MASS POP III BLACK HOLES}

Three mechanisms can create Pop III black holes during stellar collapse. In order of increasing progenitor mass, they are fallback onto a neutron star (NS) during a supernova explosion, the direct collapse of a proto-neutron star into a $\mathrm{BH}$ without an explosion, and enclosure of the core by an event horizon without ever having attained nuclear densities (Frver et al. 2001; O'Connor \& Ott 2011).

\subsection{Pop III BH Formation}

It is generally believed that core-collapse supernova explosion energies fall with increasing progenitor mass (see Fryer 2003, for a review). At some point, the explosion is too weak to fully overcome the binding energy of the star and enough ejecta falls back onto the NS to collapse it to a black hole (Fryer et al. 1999; Zhang et al. 2008). In even more massive progenitors, the core of the star collapses to a proto-neutron star without an explosion. About $1 \mathrm{~s}$ after the onset of collapse, it gains so much additional mass that it cannot support itself and it collapses to a black hole. In the most massive stars ( $300 \mathrm{M}_{\odot}$ ) the entropy of the core becomes so high that it never reaches nuclear densities. When enough material falls into the core it is suddenly engulfed by an event horizon, forming a $\mathrm{BH}$ of $>20 \mathrm{M}_{\odot}$ (Frver et al. 2001). In general, the birth masses of Pop III BH vary from the minimum black hole mass $\left(\sim 2-3 M_{\odot}\right)$ up to the mass of the progenitor star.

\subsection{Pop III BH Kicks}

The first two formation processes can impart kicks (initial velocity pulses) to low-mass BH at birth. Kick mechanisms generally fall into two categories: ejecta-driven kicks (e.g. Scheck et al. 2006; Wongwathanarat et al. 2010) and neutrino-driven kicks (see Frver \& Kusenko 2006, and references therein). Ejecta kicks occur when low-mode instabilities erupt in the shock as it is driven outward by core bounce. They are likely seeded during collapse prior to bounce and result in explosion asymmetries that impart a net linear momentum to the neutron star (NS). Above $32 \mathrm{M}_{\odot}$ fallback is total, and no impulse is imparted to the BH. Neutrino kicks arise when magnetic field lines through the center of the star are crushed to extremely high densities during collapse, polarizing

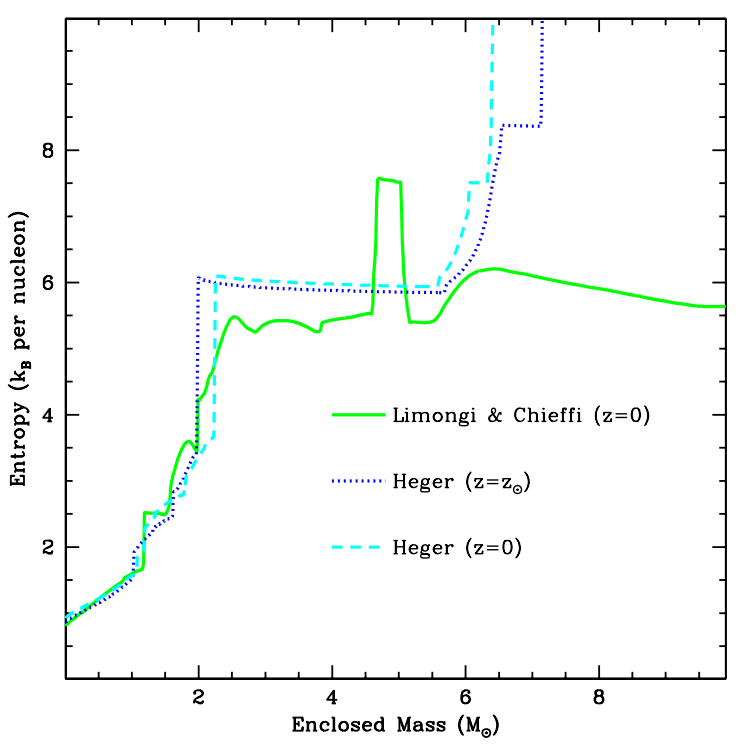

Fig. 1.- Entropy versus enclosed mass for three $25 \mathrm{M}_{\odot}$ stars. Note that the differences due to the codes are greater than those due to metallicity.

neutrinos created by the core during deleptonization and inducing anisotropies in emission that deliver an impulse to the NS. Consequently, neutrinos can impart momentum to the NS (and therefore the $\mathrm{BH}$ ) even if there is no explosion.

Pop III core-collapse SNe imparted kicks to neutron stars and black holes in the same manner as in the Galaxy today. Both kick mechanisms arise from asymmetries in the explosion engine that are determined by the structure of the inner core (inner $3-4 \mathrm{M}_{\odot}$ ) of the star. We show the entropy profile of this core for three $25 \mathrm{M}_{\odot}$ stars at collapse in Figure 1, a zero metallicity star modeled by Chieffi \& Limongi (2004) which collapsed with a mass of $24.7 \mathrm{M}_{\odot}$, a zero metallicity star modeled by Woosley \& Heger (2007) which collapsed with a mass of $24.9 \mathrm{M}_{\odot}$ and a solar metallicity star modeled by Wooslev \& Heger (2007) which collapsed with a mass of $12.9 \mathrm{M}_{\odot}$. Since metallicity has very little effect on the structure below $6 \mathrm{M}_{\odot}$, the engine will not differ significantly between a zero and solar metallicity star and they will exhibit similar kick distributions. The structures of the cores of very massive stars do change with metallicity but we do not expect kicks in their supernovae.

\section{POST-SUPERNOVA KINEMATICS OF LOW-MASS POP III BH}

Fully developed models for kick mechanisms do not yet exist, so neither the number of Pop III seed BH kicks nor their velocity distributions can be calculated from first principles. However, natal kicks are commonly observed in compact remnants in the Galaxy today and there are models that infer reasonable relationships between $\mathrm{BH}$ and NS kick distributions, which have been measured for a large sample of pulsars. In our study we adopt the pulsar velocity distribution of Arzoumanian et al. (2002). It is bimodal, with each mode being described by a Maxwellian: $40 \%$ have a dispersion of $90 \mathrm{~km} / \mathrm{s}$ and $60 \%$ have a dispersion of $500 \mathrm{~km} \mathrm{~s}^{-1}$. We derive velocity distributions for low-mass Pop III BH by assuming that in both mechanisms the black hole simply inherits the 

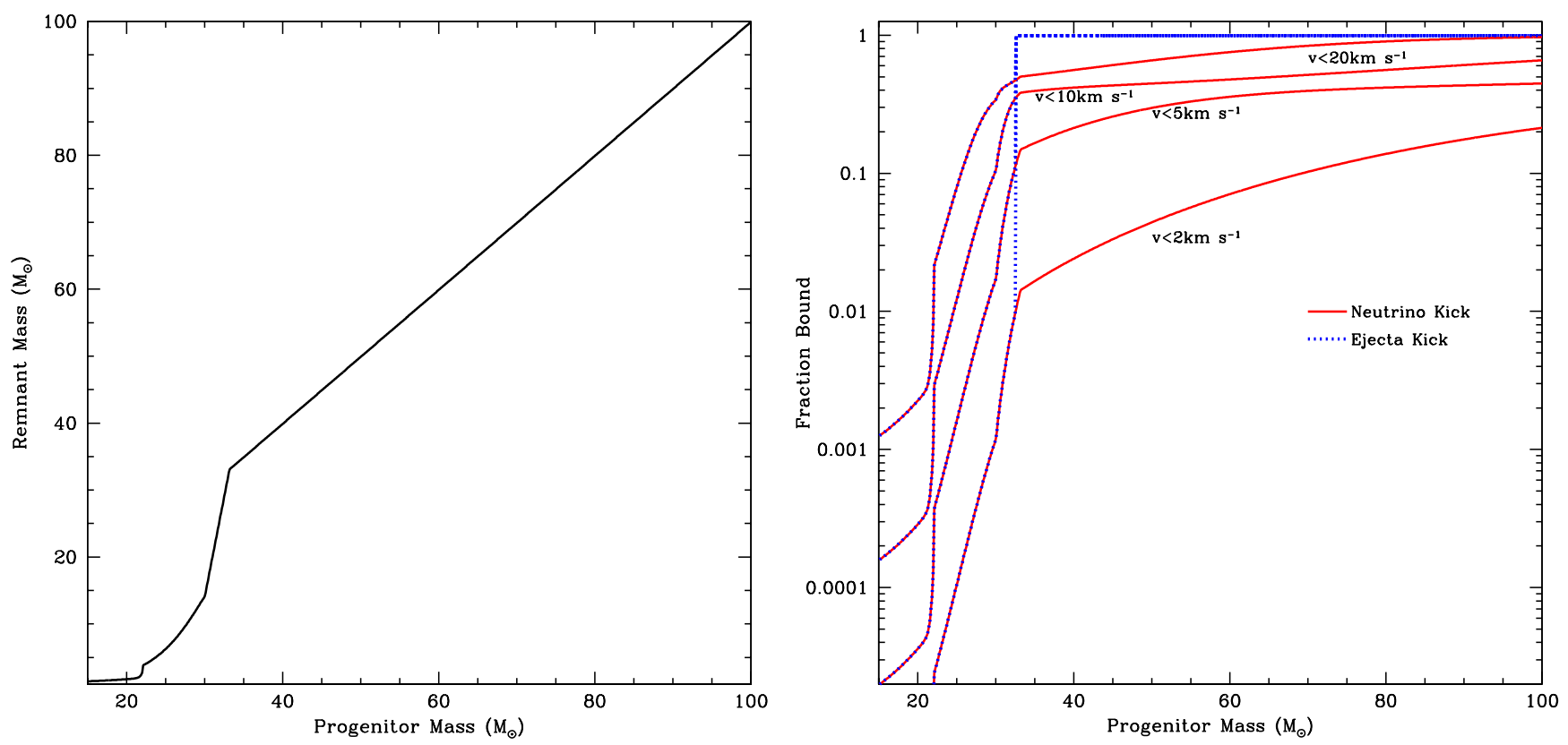

Fig. 2.- Statistical properties of low-mass Pop III BH at birth. Left: black hole mass as a function of progenitor mass. Right: BH retention fraction in the halo as a function of progenitor mass.

linear momentum of the NS:

$$
v_{B H}=v_{N S} \frac{m_{N S}}{m_{B H}},
$$

where $m_{N S}$ is the Chandrasekar mass, $1.4 \mathrm{M}_{\odot}$. Consequently, the $\mathrm{BH}$ kick velocity is inversely proportional to its mass. We derive our $\mathrm{BH}$ mass distribution from the latest estimates of Fryer \& Heger (2011) for zerometallicity stars, assuming rapid explosions. We show the distribution for these new fits in the left panel of Figure 2 In reality, the $\mathrm{BH}$ could acquire more momentum than the NS intermediary because of the tendency of weak explosions to be more delayed, which allows lowmode instabilities additional time to develop and create greater asymmetry in the ejecta (Frver \& Heger 2011). With our black hole mass and pulsar velocity distributions we can estimate the retention fraction of $\mathrm{BH}$ in halos as a function of progenitor mass for a variety of escape velocities from the halo, as we show in the right panel of Figure 2. Above $\sim 32 M_{\odot}$, the kick velocity drops to zero for the ejecta mechanism and we expect full retention for stars above this mass limit in the absence of neutrino kicks. If there are neutrino kicks, retention fractions for $\mathrm{BH}$ below $40 \mathrm{M}_{\odot}$ are less than $10 \%$ in the halos in which most Pop III stars form (those with $v_{\text {esc }}<$ $5 \mathrm{~km} / \mathrm{s}$ ), and fall below $1 \%$ for BH below $32 \mathrm{M}_{\odot}$.

\section{DISCUSSION AND CONCLUSION}

The number of stars that $10^{5}-10^{7} \mathrm{M}_{\odot}$ halos typically form is not well constrained. The studies of Pop III protostellar disk fragmentation performed thus far do not follow the evolution of the disk for enough dynamical times to determine the ultimate fate of the fragments, which may later merge with the central object or be destroyed by gravitational torques before becoming distinct stars. Ionizing UV radiation from one star-forming frag- ment or even from a nearby halo can also prematurely halt the collapse of other fragments in the disk, lowering the number of stars that eventually form in the halo (e.g. Susa \& Umemura 2006; Whalen et al. 2008a; Susa et al. 2009; Whalen et al. 2010). We also note that while the evolution of the fragments in the disk is expected to be roughly coeval, their 5 - 10 Myr quasistatic collapse times raise the possibility that the first star to form in the halo may explode and pre-empt the collapse of other fragments (e.g. Sakuma \& Susa 2009). Consequently, the number of low-mass Pop III stars that occupy the halo likely ranges from one to at most ten.

Ejecta-driven natal kicks will evict most $20-32 \mathrm{M}_{\odot}$ $\mathrm{BH}$ from their host halos, neutrino-driven kicks can drive more than $90 \%$ of $32-40 \mathrm{M}_{\odot} \mathrm{BH}$ from their halos, as we show in the right panel of Figure 2. This guarantees that on average all the $\mathrm{BH}$ will vacate the halo even if ten stars originally formed in it. Post-supernova kinematics thus strongly discourages $20-40 \mathrm{M}_{\odot}$ Pop III BH from becoming supermassive because they are ejected from their fuel supply and deprived of crucial early e-foldings in mass. This process greatly reduces the parameter space in stellar mass from which SMBH can originate (e.g. Tanaka \& Haiman 2009; Lippai et al. 2009), especially if Pop III stars were mostly less than $50 \mathrm{M}_{\odot}$. Also, if a given halo is capable of supporting early continuous Eddington rate accretion, a $20-40 \mathrm{M}_{\odot} \mathrm{BH}$ is much less likely to become supermassive than a $100 \mathrm{M}_{\odot} \mathrm{BH}$, either because it is ejected from the halo at birth or because it must undergo additional e-folding times to reach large masses.

If most low-mass Pop III black holes were ejected from their halos at $z \sim 20$, where are they today? If on average they depart their host halos at $\sim 500 \mathrm{~km} / \mathrm{s}$, they are unlikely to encounter another halo capable of capturing them in less than a Hubble time, and so many 
of these $\mathrm{BH}$ were exiled to the voids between galaxies. Over time, they may have gradually gained mass as they encountered high-density regions. In contrast, Pop III $\mathrm{BH}$ above $40 \mathrm{M}_{\odot}$ are unlikely to be born with kicks and remain in the halo, intermittently accreting and growing over cosmic time. These black holes are much more likely to reside in the galaxies into which their host halos were taken, a few of which may have become the supermassive black holes found in the SDSS quasars today.
We thank the anonymous referee for comments that improved the quality of this paper and Jarrett Johnson and Brian O'Shea for valuable comments. DJW was supported by the Bruce and Astrid McWilliams Center for Cosmology at Carnegie Mellon University. Work at LANL was done under the auspices of the National Nuclear Security Administration of the U.S. Department of Energy at Los Alamos National Laboratory under Contract No. DE-AC52-06NA25396.

\section{REFERENCES}

Abel, T., Bryan, G. L., \& Norman, M. L. 2002, Science, 295, 93

Alvarez, M. A., Wise, J. H., \& Abel, T. 2009, ApJ, 701, L133

Arzoumanian, Z., Chernoff, D. F., \& Cordes, J. M. 2002, ApJ, 568,289

Bromm, V., Coppi, P. S., \& Larson, R. B. 2002, ApJ, 564, 23

Chieffi, A., \& Limongi, M. 2004, ApJ, 608, 405

Clark, P. C., Glover, S. C. O., Smith, R. J., Greif, T. H., Klessen, R. S., \& Bromm, V. 2011, Science, 331, 1040

Djorgovski, S. G., Volonteri, M., Springel, V., Bromm, V., \& Meylan, G. 2008, in The Eleventh Marcel Grossmann Meeting On Recent Developments in Theoretical and Experimental General Relativity, Gravitation and Relativistic Field Theories, ed. H. Kleinert, R. T. Jantzen, \& R. Ruffini, 340-367

Fryer, C. L. 2003, International Journal of Modern Physics D, 12 , 1795

Fryer, C. L., Colgate, S. A., \& Pinto, P. A. 1999, ApJ, 511, 885

Fryer, C. L. \& Heger, A. 2011, Astronomische Nachrichten, 332, 408

Fryer, C. L. \& Kusenko, A. 2006, ApJS, 163, 335

Fryer, C. L., Woosley, S. E., \& Heger, A. 2001, ApJ, 550, 372

Greif, T. H., Springel, V., White, S. D. M., et al. 2011, ApJ, 737, 75

Greif, T. H., Johnson, J. L., Bromm, V., \& Klessen, R. S. 2007, ApJ, 670,

Heger, A. \& Woosley, S. E. 2002, ApJ, 567, 532

Hosokawa, T., Omukai, K., Yoshida, N., \& Yorke, H. W. 2011, Science, 334, 1250

Joggerst, C. C., Almgren, A., Bell, J., Heger, A., Whalen, D., \& Woosley, S. E. 2010, ApJ, 709, 11

Johnson, J. L., Khochfar, S., Greif, T. H., \& Durier, F. 2011, MNRAS, 410, 919

Karlsson, T., Johnson, J. L., \& Bromm, V. 2008, ApJ, 679, 6

Kitayama, T. \& Yoshida, N. 2005, ApJ, 630, 675

Kitayama, T., Yoshida, N., Susa, H., \& Umemura, M. 2004, ApJ, 613,631

Li, Y. 2011, ArXiv e-prints

Lippai, Z., Frei, Z., \& Haiman, Z. 2009, ApJ, 701, 360
Milosavljević, M., Bromm, V., Couch, S. M., \& Oh, S. P. 2009, ApJ, 698, 766

Mortlock, D. J., Warren, S. J., Venemans, B. P., Patel, M., Hewett, P. C., McMahon, R. G., Simpson, C., Theuns, T., Gonzáles-Solares, E. A., Adamson, A., Dye, S., Hambly, N. C., Hirst, P., Irwin, M. J., Kuiper, E., Lawrence, A., \& Röttgering, H. J. A. 2011, Nature, 474, 616

O'Connor, E., \& Ott, C. D. 2011, ApJ, 730, 70

O'Shea, B. W. \& Norman, M. L. 2007, ApJ, 654, 66

Park, K., \& Ricotti, M. 2012, ApJ, 739, 2

Park, K., \& Ricotti, M. 2012, ApJ, 747, 9

Regan, J. A. \& Haehnelt, M. G. 2009, MNRAS, 396, 343

Sakuma, M. \& Susa, H. 2009, ApJ, 698, 155

Scheck, L., Kifonidis, K., Janka, H., \& Müller, E. 2006, A\&A, 457, 963

Shang, C., Bryan, G. L., \& Haiman, Z. 2010, MNRAS, 402, 1249

Smith, R. J., Glover, S. C. O., Clark, P. C., Greif, T., \& Klessen, R. S. 2011, MNRAS, 414, 3633

Stacy, A., Greif, T. H., \& Bromm, V. 2010, MNRAS, 403, 45

—. 2012, MNRAS422, 290

Susa, H. \& Umemura, M. 2006, ApJ, 645, L93

Susa, H., Umemura, M., \& Hasegawa, K. 2009, ApJ, 702, 480

Tanaka, T. \& Haiman, Z. 2009, ApJ, 696, 1798

Turk, M. J., Abel, T., \& O'Shea, B. 2009, Science, 325, 601

Whalen, D., Abel, T., \& Norman, M. L. 2004, ApJ, 610, 14

Whalen, D., Hueckstaedt, R. M., \& McConkie, T. O. 2010, ApJ, 712,101

Whalen, D., O'Shea, B. W., Smidt, J., \& Norman, M. L. 2008a, ApJ, 679, 925

Whalen, D., van Veelen, B., O'Shea, B. W., \& Norman, M. L. 2008b, ApJ, 682, 49

Wise, J. H., Turk, M. J., \& Abel, T. 2008, ApJ, 682, 745

Wongwathanarat, A., Janka, H., \& Müller, E. 2010, ApJ, 725, L106

Woosley, S. E., \& Heger, A. 2007, Phys. Rep., 442, 269

Yoshida, N., Oh, S. P., Kitayama, T., \& Hernquist, L. 2007, ApJ, 663,687

Zhang, W., Woosley, S. E., \& Heger, A. 2008, ApJ, 679, 639 